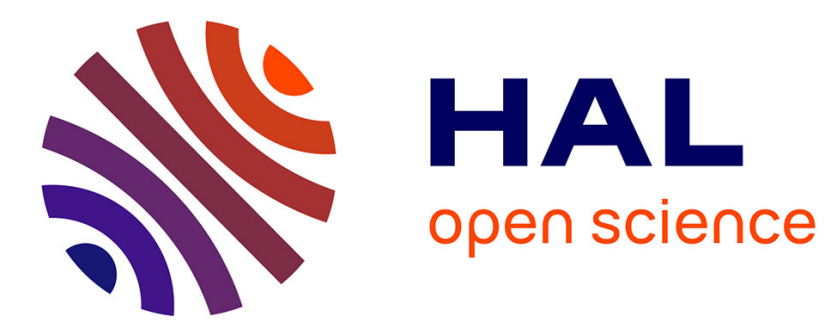

\title{
Long term analysis of time series of satellite images
}

Thomas Guyet, Hervé Nicolas

\section{To cite this version:}

Thomas Guyet, Hervé Nicolas. Long term analysis of time series of satellite images. Pattern Recognition Letters, 2015, 70, pp.17-23. 10.1016/j.patrec.2015.11.005 . hal-01239504

\section{HAL Id: hal-01239504 https://hal.inria.fr/hal-01239504}

Submitted on 8 Dec 2015

HAL is a multi-disciplinary open access archive for the deposit and dissemination of scientific research documents, whether they are published or not. The documents may come from teaching and research institutions in France or abroad, or from public or private research centers.

$$
\text { Copyright }
$$

L'archive ouverte pluridisciplinaire HAL, est destinée au dépôt et à la diffusion de documents scientifiques de niveau recherche, publiés ou non, émanant des établissements d'enseignement et de recherche français ou étrangers, des laboratoires publics ou privés. 


\title{
Long term analysis of time series of satellite images
}

\author{
Thomas Guyet ${ }^{\mathrm{a}}$, Hervé Nicolas ${ }^{\mathrm{b}}$ \\ ${ }^{a}$ AGROCAMPUS-OUEST/IRISA, UMR 6074 \\ 65 rue de Saint-Brieuc, CS 84215, 35042 Rennes, FRANCE \\ ${ }^{b}$ AGROCAMPUS-OUEST/INRA, UMR1069 Soil Agro and hydrosysteme Spatialisation \\ 65 rue de Saint-Brieuc, CS 84215, 35042 Rennes, FRANCE
}

\begin{abstract}
Satellite images allow the acquisition of large-scale ground vegetation. Images are available along several years with a high acquisition rate. Such data are called satellite image time series (SITS). We present a method to analyse an SITS through the characterization of the evolution of a vegetation index (NDVI) at two scales: annual and multi-annual. We evaluate our method on SITS of the Senegal from 2001 to 2008 and we compare our method to a clustering of long time series. The results show that our method better discriminates regions in the median zone of Senegal and locates fine interesting areas.
\end{abstract}

(c) 2015 Elsevier Ltd. All rights reserved.

\section{Introduction}

Remote sensing images are broadly used to map the land cover. The land cover describes the physical material at the surface of the land (soils and vegetation). In West African countries, such information is useful to agricultural managers in order to prevent the socio-economic consequences of drought, climate changes or changes in the agricultural practices. Satellite images provide a large amount of data at a large spatial scale and at several dates. They can be analysed to map the land cover or to determine the covers change. The mapping task is mainly a classification of the pixels - the location - into categories of land covers. If the mapping categories are not known a priori, it is performed by unsupervised classification (clustering). The more discriminable are the categories, the more accurate will be the classification. One of the main problem is to provide pixel data that will easily discriminates the categories.

The effectiveness of the image classification are best demonstrated through the use of many images of the same region acquired over time (Campbell, 2002). A lot of studies have shown the interest of such multi-temporal satellite images to improve the accuracy of the classification of several classes of vegetation (Sabour et al. 2008; Petitjean et al., 2012). In fact, different types of vegetation have different phenologies, i.e. different life cycles.As a consequence, the vegetations are more easily discriminable by using multi-temporal satellite images than by analysing a single image. 
Since the two last decades, satellites acquired a global coverage of the earth with a short revisit time. The two satellites of the MODIS ${ }^{1}$ program are viewing the entire Earth's surface every 1 to 2 days. Moreover, upcoming satellites (e.g. ESA's Sentinel program) will improve both their spatial resolution and their temporal resolution, i.e. the inverse of the time to revisit the same location. Such collection of satellite images for the same region at different dates are called Satellite Image Time Series (SITS). SITS can be seen as a stack of images (one layer per date) or as a grid of time series: each pixel is associated with a time series. The Figure 1 illustrates the two views of a SITS: a spatial view, an image is a stack of images - a temporal view, an image is an array of time series. In the following, we adopt the second view. Historical data from remote sensing is often valuable because it may provide only long-term data for a large extent of geography. These data can improve our understanding of global dynamics and processes occurring on the surface. At the same time, the data is often complex to interpret.

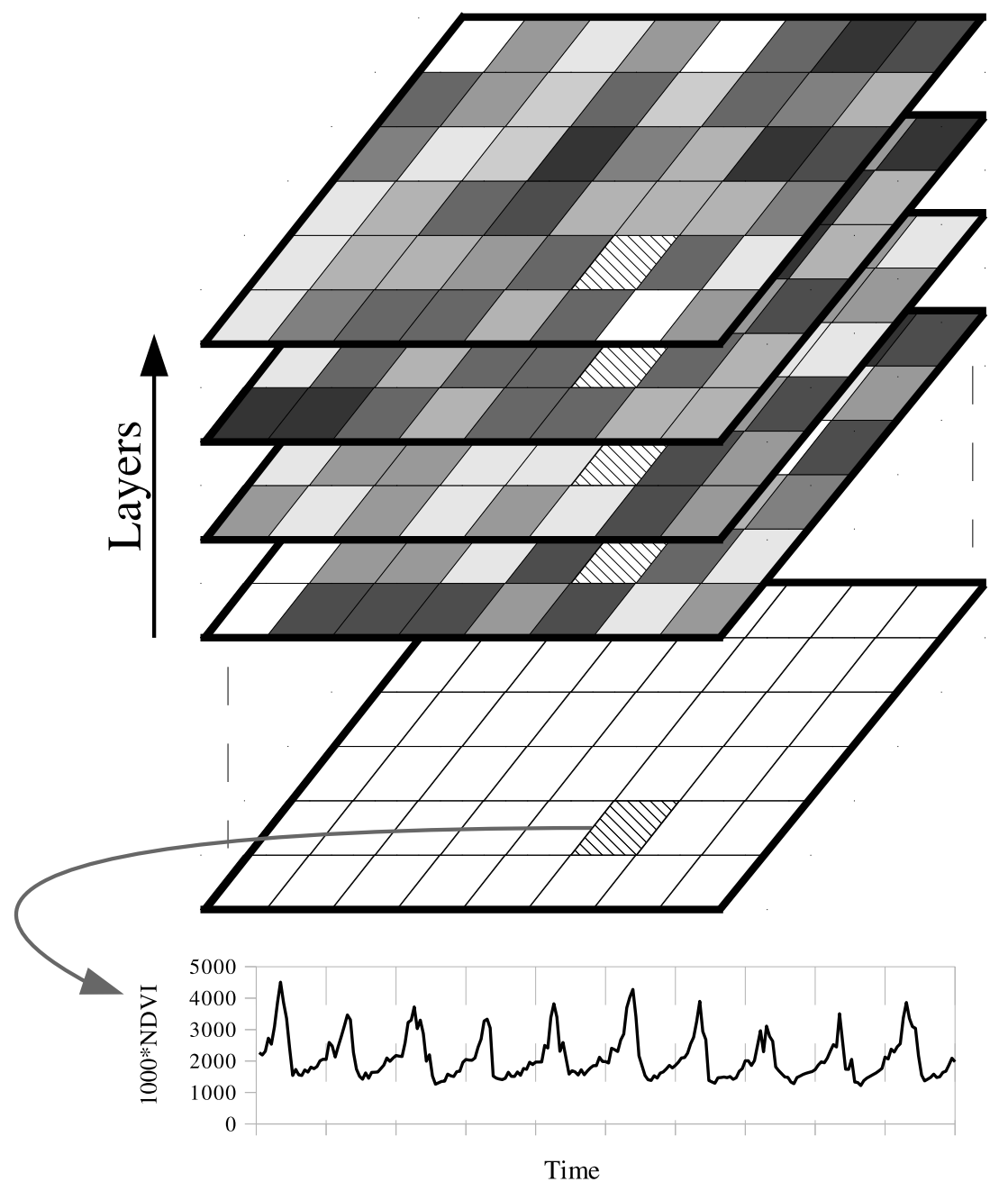

Fig. 1. Time series of satellite images (SITS). Above, vision in the form of layers of image ; Below, transverse view of a pixel in the form of a temporal series (10 years of 23 dates).

${ }^{1}$ MODIS: Moderate Resolution Imaging Spectroradiometer. 


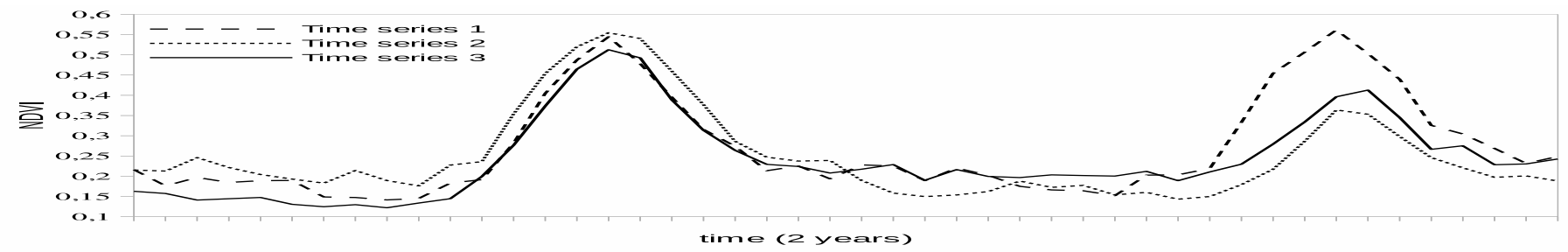

Fig. 2. Intra-annual cumulative differences hide the structural differences between time series. In the Figure, Euclidean distance between time series 2 and 3 is exactly the same than the distance between time series 1 and 3. Nonetheless, from the expert point of view, time series 2 and 3 are similar, and both dissimilar with 3 (simulated NDVI time series).

Efficient tools are required to support the analysis of such complex and massive data, in their whole temporal dimension (Kuenzer et al., 2015). We are especially interested in long-term SITS, i.e. SITS which have several intrinsically temporal-scales, for instance annual and multi-annual scales. The long-term data is useful to refine the mapping of vegetation by the characterisation of their dynamic. The evolution of the vegetation with respect to some specific events, such as drought, or to the climate change, highlights relative behavioural differences. The localization of such differences is interesting to improve the soil and water management.

The challenge of long-term analysis is to deal with the non-stationarity of the NDVI time series. Forkel et al. (2013) have pointed out the effects of inter-annual variability in NDVI time series. The distance between two long time series is disturbed by the cumulative sum of small instantaneous differences.

We illustrate this difficulty with the simulated time series of Figure 2 Considering three simulated two years $N D V I$ time series, the whole time series have the same behaviour along the first year (relatively high $N D V I$ value along the wet season), while time series 2 and 3 have a behaviour different from the time series 1 along the second year (relatively low $N D V I$ values along the wet season for time series 2 and 3). Nonetheless, Euclidean distances between pairs of time series are exactly the same. From the expert point of view, time series 2 and 3 are more similar than time series 1 and 3 .

The change of annual profile is more important than small intra-annual evolutions. A two time-scales approach can take it into account: labelling each year with a symbol that characterize the annual profile eliminates the intra-annual noise in time series. The time series 2 and 3 would be abstracted by a sequence $a_{1}>a_{1}$ (a year of type $a_{1}$ following by another year of type $a_{1}$ ) while time series 1 would be abstracted by a sequence $a_{1}>a_{2}$, where $a_{1}$ and $a_{2}$ designate a year with respectively a high or a low $N D V I$ value in wet season. In this example, the distance between time series 2 and 3 would be null while the distance between time series 1 and 3 would be not null.

Our approach is based on a two-stages temporal abstraction of time series. The two temporal scales are 1) the annual scale which is related to a vegetation cycle and 2) the multi-annual scale. At the first stage, temporal abstraction of time series consists in transforming a time series into a sequence of symbols. Each symbol corresponds to a typical annual profile of NDVI. This 
step reduces the data dimensionality and it reduces the inter-annual noise disturbance. The second stage consists in characterizing sequences of symbols to highlight multi-annual behaviours.

\section{Related works}

STIS have been used in many agronomic applications, for instance, monitoring the vegetation dynamics, monitoring the surface expansion of forests or the urban-sprawl, detecting abrupt changes like surface deformation or analysing long-term effects of climate change.

Measuring the distance between the time series remains the main challenge for clustering or segmenting SITS. Lhermitte et al. (2011) details some time series characteristics and proposes a comparison of time series similarity measures for classification and change detection of ecosystem dynamics. A large number of dissimilarities between time series have been proposed. The Euclidean distance is often used but some applications require to highlight more specific characteristics in time series. In the field of data mining, elastic measures have been introduced to compare time series (e.g. DTW (Sakoe and Chiba, 1978), LCSS (Das et al. 1997) or MVM (Latecki et al., 2005)). These dissimilarities take into account the time warping where Euclidean distance focus attention on the date of the changes in time series. Ketterlin and Gancarski (2007) proposed to use such elastic distance to cluster the pixels of multi-date images with presence of clouds (missing values in time series). Then, Petitjean et al. (2011) proposed the DBA algorithm, a modified K-Means algorithm to cluster time-series using the DTW, and applied it to SITS.

If SITS are made of sequences of symbolic values, the classical time series measure may be applied, but sequential pattern mining techniques may be also used. Julea et al.(2011) present a technique to support experts in agricultural monitoring, by mining SITS over cultivated areas. Their approach extends sequential patterns in order to extract sets of connected pixels sharing a similar temporal evolution. Sequential pattern mining algorithms extract the frequents behaviours. As a consequence, a pixel may not contain any of the frequent sequences and it may not be assigned to any cluster. On the opposite, a pixel may contain several frequent sequences, and it is difficult to assign a single cluster to the pixel.

All these methods have been applied on short time-series and previously noticed, the non-stationary of the NDVI time series raises problems the cluster long time series. Both time-series distances approaches and symbolic sequences approach can not solve them. Practically, only few works are focused on long-term SITS (multi-annual SITS) or deal with multi-temporal scales SITS. Bradley et al. (2007) proposed to fit spline-based curves and inter-annual curve on time series to identifies phenological shape. The author explains that this may lead to improved land cover classification, but they do not explain how to do it. The method BFAST (Verbesselt et al. 2010) uses a Fourier decomposition to take into account the changes and the seasonal trends to improve the accuracy of failure-detection methods change in the presence of climate variations. This method can be used to detect long-term phenological changes within time series by analysing the seasonal component of time series for changes in phenology. 
Campo-Bescos et al. (2013) applied Dynamic Factor Analysis (DFA), a multivariate times series dimension reduction technique, to investigate the dynamics of vegetation coverage across two long time scales (2001-2010 and 1982-2010). Thus, they identify the most important physical drivers of vegetation cover in large watersheds in southern Africa. Nonetheless, these two methods are used to create maps that localize abrupt changes but do not provide a map of land-cover.

All previously presented approaches are working on optical images. In remote sensing, SAR (Synthetic Aperture Radar) images are often used to monitor surface deformation Ferretti et al. (2001) because there are cloud-free. SAR images analysis are based on interferometry which enables the extraction of characteristics from the radar signals. Nonetheless, distinguishing vegetation from radar signal is still a huge challenge. For this reason, SAR images are not widely used for vegetation mapping.

The other main satellite image processing task is the segmentation. The objective of the segmentation is to determine the homogeneous regions, corresponding to different characteristics of the land-cover. The presented method does not segment the SITS. Any segmentation could be used as a post-processing. Petitjean and Weber (2014) recently proposed to use Quasi-Flat Zone segmentation algorithm in combination with time series clustering with DTW to improve the computing time efficiency. Petitjean et al. (2012) proposed to use a segmentation step to provide contextual information about pixels of a SITS. In their method, the authors segment each image and then, use a clustering to characterize each pixel, defined by the time series of the values of its segment. Lhermitte et al. (2008) introduced a multi-temporal hierarchical image segmentation (MTHIS) methodology to generate a hierarchical set of segments based on spatial similarity of remote sensing time series. The principle of MTHIS is analogous to classical hierarchical image segmentation which uses bottom-up region-merging technique. They use a dissimilarity criterion based on the components of a Fourier Transform.

Finally, numerous works have been proposed to analyse video sequences and recent works have been developed to segment videos with spatio-temporal approaches. These methods could be applied to SITS. We illustrate this by two recent references working on long-term temporal interactions among objects in the video and that are closed to our approach. Lezama et al. (2011) proposed an efficient spatio-temporal video segmentation algorithm, which naturally incorporates long-range motion cues. Trichet and Nevatia (2013) introduce spatio-temporal tubes and supertubes. The tubes segment a video based on clusters of the dense trajectory.

\section{Methodology for long-term SITS analysis}

In this section, we present the proposed methodology to analyse long-term dynamics of land-cover. Long-term means that we analyse the behaviour of land-cover over several years. As the land covers dynamic is driven by the annual scale and the multi-annual scale, our method has two main steps illustrated in Figure 3 


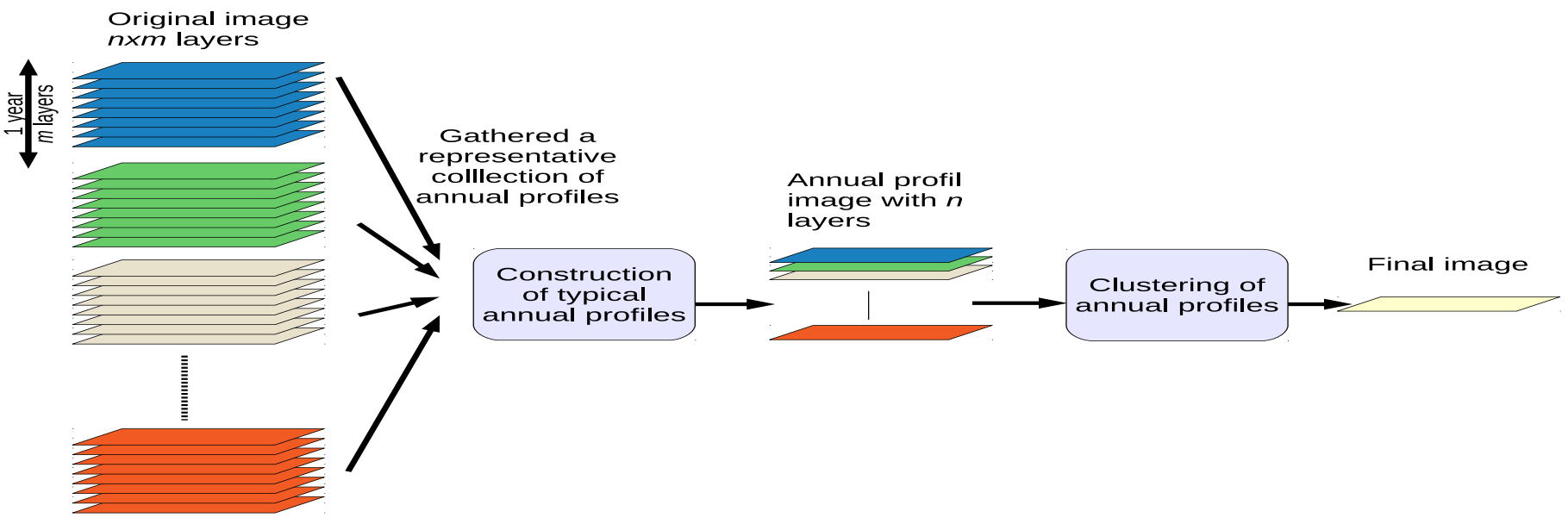

Fig. 3. Illustration of the proposed methodology.

- the characterisation of annual profiles from a set of annual SITS. This step leads to the construction of typical annual images that characterize the behaviour of a pixel location along a specific year,

- the characterisation of the sequence of annual images. This step leads to the construction of a single image that sum up the behaviour of a pixel location along the long-term period.

In the sequel, we use the following notations: $\mathcal{I}=\left(I_{i}^{j}\right)_{i=1 . . n, j=1 . . m}$ is the SITS made of $n \times m$ images where $n$ is the number of years and $m$ is the number of images per year. As a consequence, $I_{i}^{j}$ denotes the $j$-th image of the $i$-th year, and $I_{i}^{j}(x, y)$ is the pixel located at the coordinates $(x, y)$ of the layer. A pixel is described by a real value, i.e. $I_{i}^{j}(x, y) \in \mathbb{R}$. We also denote by $I_{i}(x, y) \in \mathbb{R}^{m}$ the time series that describes the dynamic at location $(x, y)$ during the year $i$. Such time series are called annual profiles.

We can notice that in the general case, satellite images have several bands (i.e. $I_{i}^{j}(x, y) \in \mathbb{R}^{\beta}$, where $\beta$ is the number of bands). It can typically be spectral bands or index bands (e.g. NDVI, EVI) constructed by composing the spectral values. For sake of simplicity, we present our methodology considering that each satellite image has a single band, typically a vegetation index. It can easily be generalised considering that the distance between two pixels at time $t$ would be computed by an Euclidean distance in $\mathbb{R}^{\beta}$.

\subsection{Construction of the image of typical annual profiles}

The construction of the image of typical annual profiles is described in the algorithm 1 This algorithm has two steps: firstly, the extraction of typical annual profiles and, secondly, the construction of the image stack: each layer of the image gives the index of the closest typical annual profiles to the annual profile of each pixel.

The first step constructs a set of typical annual profiles $\left(\mathcal{P}=\left\{p 1, \ldots, p_{k}\right\}\right)$. A clustering algorithm identifies the most significant clusters in the set of the time series (annual profiles), then typical annual profiles are extracted from the clusters of similar times series. 
In our long-term SITS, we have several years of data per pixel. The method compares images of several years with each other. It implies that same clusters must be shared by all the years to enable their comparison. In such objective, the set $E$ is made of annual profiles that come from all years of the original SITS $\mathcal{I}$. Dealing with large images along several years leads to a possible huge set $E$. To tackle the issues of memory requirement or of computation time, we sample the dataset by selecting a subset of annual profiles to cluster. This sampling is ensured by function $E c h(\mathcal{I}, i)$. Several sampling strategies can be applied, for instance a regular sampling (regular in space or/and in time). A possible alternative is a sampling that gives a high probability to preselected regions or/and years that must be clustered more carefully. This function has to be define with respect to the objective, to the dataset and to the computing resources. In Section 4 , we use a regular sampling.

For the clustering algorithm, the most important is to select the distance that will give the most significant results. We selected the Euclidean distance for its ability to compare the date of the bloom start. On the other hand, Euclidean distance can be used with a large number of clustering algorithm while dissimilarities have to be used carefully. The Clustering function can be choose amongst classical clustering algorithms (K-Means, EM, etc.). In Section 4, we use a K-Means algorithm.

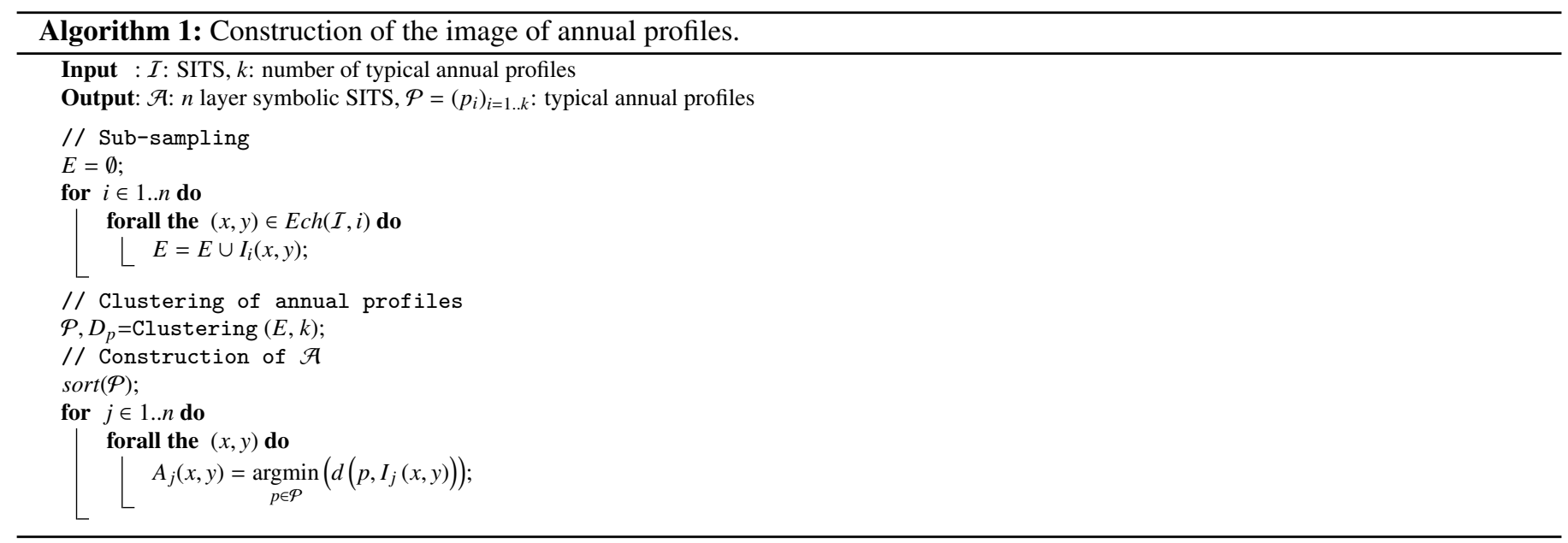

Once the typical annual profiles have been constructed, the image of annual profiles, $\mathcal{A}$, is constructed. For each year $j$, the best typical annual profiles is assigned to all the pixel of $A_{i}$.

Beforehand, typical annual profiles have been sorted. $p_{1}$ is the typical annual profiles with the lower mean value, i.e. a dry year. $p_{2}$ is the closest typical annual profile from $p_{1}$, etc. This step order approximatively the typical annual profiles from the dryer to the more wet year. Note that it does not generate a linear order. It does not ensure that, for instance, $p_{2}$ is closer to $p_{5}$ than it is to $p_{8}$. This ordering is useful to interpret images but it is not required in the second step of our methodology.

\subsection{Construction of sequences of classes}

Each pixel $(x, y)$ of image $\mathcal{A}$ is defined by a symbolic sequence, denoted by $\mathcal{A}(x, y)=\left(a_{i}\right)_{i \in 1 . . n}$, where $n$ is the length of the sequence and for all $i, a_{i}$ is the identifier of a typical annual profile, $a_{i} \in \mathcal{P}$. The construction of sequence clusters consists in 
identifying the most similar sequences between them. The distance between two sequences $a=\left(a_{i}\right)_{i \in 1 . . n}$ and $b=\left(b_{i}\right)_{i \in 1 . . n}$ is defined by:

$$
\forall(i, j), d_{s}(a, b)=\sqrt{\sum_{u=1}^{n} D_{p}\left(a_{u}, b_{u}\right)^{2}}
$$

where $D_{p}$ is a distance matrix constructed by the Euclidean distance between the typical annual profiles.

The KMeans algorithm can not be used to cluster symbolic sequences (Diday and Murty, 2005). KMeans algorithm requires the computation of centroids as the barycentre of clusters elements. But the barycentre of symbolic sequences has no meaning. To tackle this issue, we propose to use the KMedoid clustering algorithm (Kaufman and Rousseeuw, 1990). This algorithm uses instances as prototypes of the clusters, thus there is no centroid to compute.

The KMedoid algorithm does not require to compute the distance matrix but it requires a true distance, including a triangular inequality property, to ensure the convergence. The measure $d_{s}$ we defined above is a distance and the KMedoids algorithm can be used to cluster the symbolic sequences.

Each cluster $C_{i}, i \in 1 . . l$ is represented by its set of sequences. The final image, $\mathcal{S}$, is an image with one layer that holds the identifier of the cluster of each pixel:

$$
\forall x, y, \mathcal{S}(x, y)=\underset{i \in\{1 \ldots l\}}{\operatorname{argmin}}\left(d_{s}\left(C_{i}, \mathcal{A}(x, y)\right)\right)
$$

\section{Experimental results on Senegal images}

Agricultural sustainability has the highest priority in all countries, whether developed or in developing. Drought is a one of the major issue in African countries especially in Senegal. Senegal is located in a transitional zone between the rainforest (in the south) and arid zones (in the north). Land cover characterization and mapping through remote sensing is one of the major perspectives for supporting sustainable agricultural management and development.

The Normalized Difference Vegetation Index, or NDVI, is a remote sensing-based indicator used for drought detection and monitoring (Tucker et al. 1985). NDVI is related to the rate of soil cover by vegetation. It is the vegetation index the most used by the scientific community to the study of the vegetation. $N D V I$ is defined by $N D V I=(P I R-R) /(P I R+R)$ where $P I R$ and $R$ are the reflectance $2^{2}$ respectively in the near infrared and the red. NDVI can be used to characterize the health of the vegetation there, relative to the norm. A region in which there is a period of reduced plant growth (due to a lack of precipitation) can be labelled as "in drought".

Since the two last decades, satellites acquired images continuously. The two satellites of the MODIS program are acquiring NDVI images of the entire Earth's surface every 1 to 2 days. Because of the popularity of the NDVI, the annual time series of this

\footnotetext{
${ }^{2}$ The reflectance is the proportion of incident lighting reflected by a surface. These values depends on the wavelength and on the type of ground.
} 
index have been widely studies. Their high temporal resolution offers the opportunity to define time profiles and characterize the vegetation dynamics on the basis of different temporal scales. Hence the remote sensing techniques based on time series analysis allow for characterizing the inter-annual and intra-annual variations of the vegetation canopy with a functional representation that is continuous and stable between years (Bradley et al., 2007) and, at the same time, accounting for non-stationary processes.

In this work, we were interested in characterising the vegetation dynamic to improve the drought mapping of the Senegal. We are especially interested in improving the mapping considering the resilience ability of the vegetation over several years. The vegetation devastated by drought regenerates with varying duration depending on the impact of the drought. For this reason, analysis of long-term time-series can improve mapping precision. To reflect seasonality, the approach we propose characterizes the vegetation dynamics on two temporal-scales: annual and multi-annual.

\subsection{Data and parameters}

The spatial extent of SITS includes Senegal with a medium resolution $(250 \mathrm{~m})$. The size of each image is $5137 \times 4828$. The SITS holds images along 7 years from (2001 to 2008) with 23 images per year (one image every 16 days). This 7 years period have been selected to study consequences of a major drought that appends in years 2002 and 2003. MOD13Q1 produc $]^{3}$ provides NDVI images that are a composition of the images available over a 16 days period. The composition reduces the cloud coverage and all images can be considered as cloud-free.

The default parameters of the experiments are $k=30$ (number of clusters of typical multi-annual sequences) and $l=100$ (number of typical annual profiles). For time series clustering, the $E c h$ function samples the image with 1 pixel each 20 pixels (same pixels for every images).

\subsection{Construction of typical annual profiles}

Figure 4 illustrates five clusters profiles amongst the $k=30$ profiles we obtained from annual times series. Figure 5 spatiotemporally locates these five clusters (white pixels correspond to pixels classified in one of the other 25 clusters). We focus our attention on only the four first years of the sequence around the drought of year 2002.

Cluster $C_{1}$ corresponds to the NDVI profile of water (very low reflectance all along the year). This cluster is located on rivers and lakes on each image of Figure 5. Cluster $C_{4}$ corresponds to very dry regions (few vegetation). These regions are mainly located in the south. Cluster $C_{4}$ appears in a lot of regions, mainly in the south. Clusters $C_{8}$ and $C_{11}$ are located in medium latitudes. The annual profiles show that $C_{8}$ corresponds to a dryer region than $C_{11}$ (cf. Figure 4): firstly, we notice that the vegetation bloom in $C_{8}$ is later than in $C_{11}$. Secondly, the duration of the vegetation is shorten. The spatial distribution of the two clusters is one of the

\footnotetext{
${ }^{3}$ see modis.gsfc.nasa.gov/for more details about the MODIS images characteristics.
} 


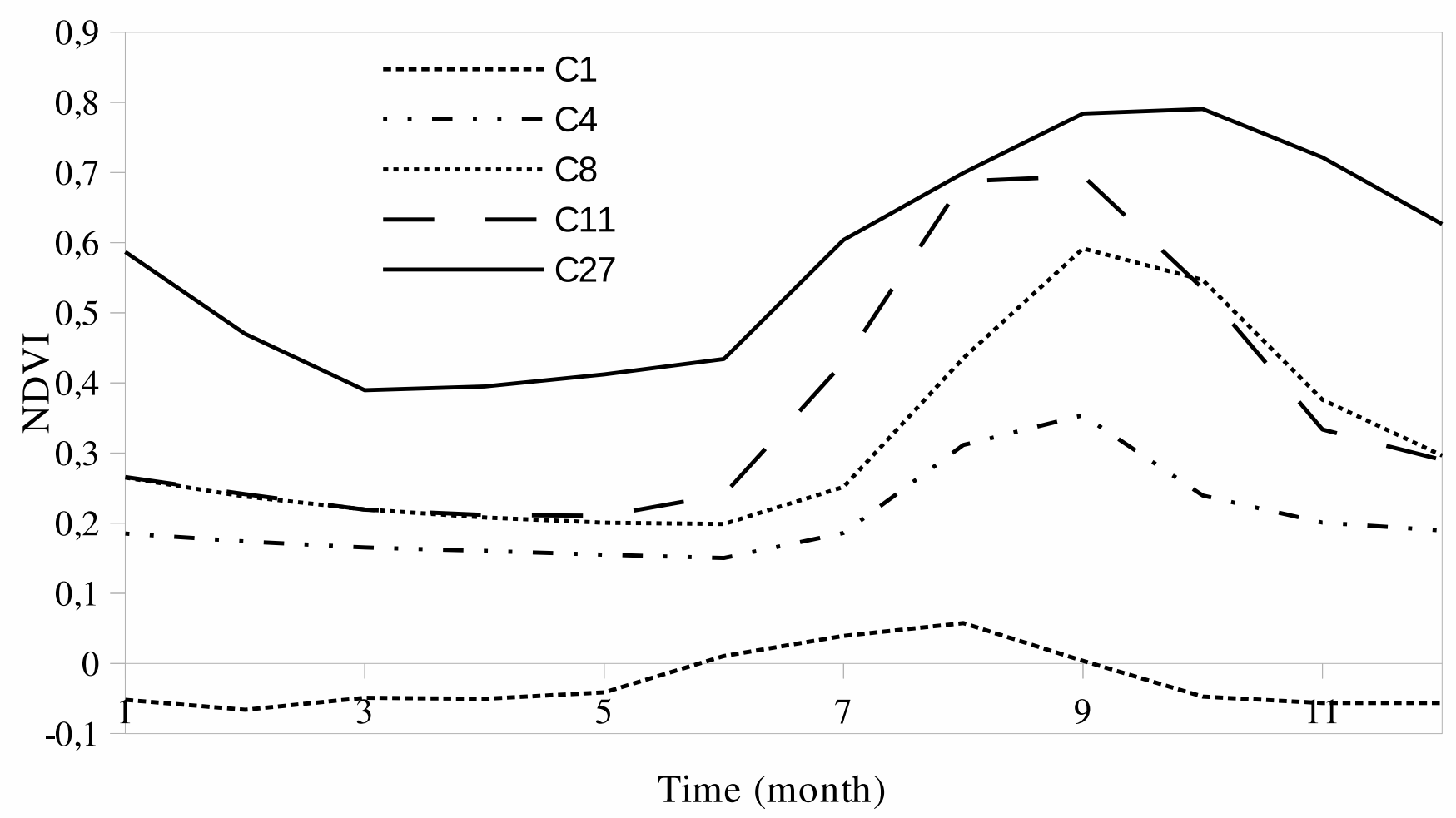

Fig. 4. Five typical annual profiles (see details in the text)
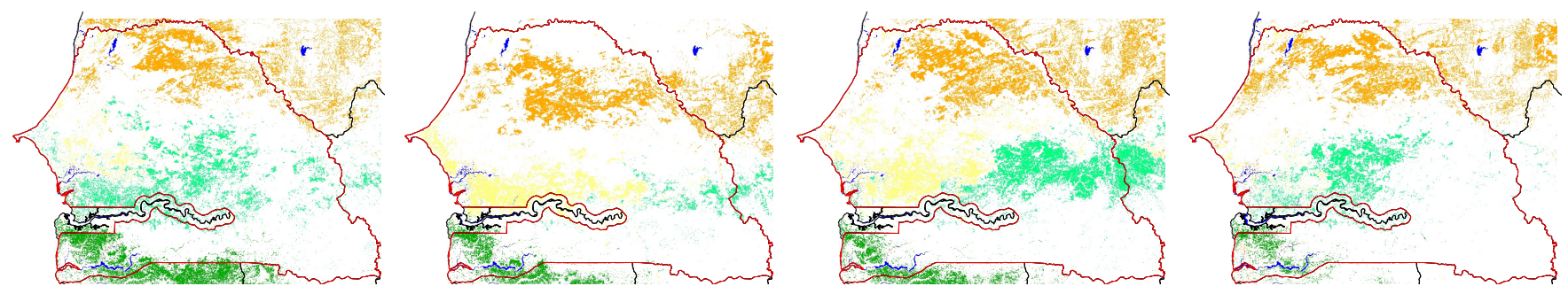

Fig. 5. Maps that localizes clusters defined by the typical annual profiles of Figure 4 in 2001 to 2004 (from left to right, then top to bottom). Blue: $C_{1}$, orange: $C_{4}$, yellow: $C_{8}$, soft green: $C_{11}$, green: $C_{27}$. Red line visualizes the administrative boundaries of Senegal.

interesting point. It changes with respect to the year, highlighting different dynamical behaviours. In 2002 and in 2004, cluster $C_{11}$ is dominant. In 2002, $C_{8}$ almost disappears to the benefit of "dryer clusters" such as $C_{11}$. In 2003, these clusters are both present in the image. One is located in the east of the Senegal while the other is present in the west. This highlights two different resilience behaviours of east and west while confronted to a drought. Finally, cluster $C_{27}$ is associated to regions with a dense vegetation. This cluster is located in Casamance (south Gambia), in the south-west of Senegal. This region is wet, made of forest and rivers (Gambia river). All along the years, the regions of this cluster are geographically stables.

One of the specificities of our method is to cluster annual profiles of all the years at the same time. Therefore, it is possible to analyse spatio-temporal phenomenons. Some clusters, such as $C_{27}$, show local dynamics (specific to a region and stable in time). While some other clusters show phenomenons that evolve in time: the migration to the south of $C_{4}$ confirms that 2002 was a particular dry year. Missing clusters are also informative. Cluster $C_{8}$ is present only in 2002 and 2003. This cluster is therefore 

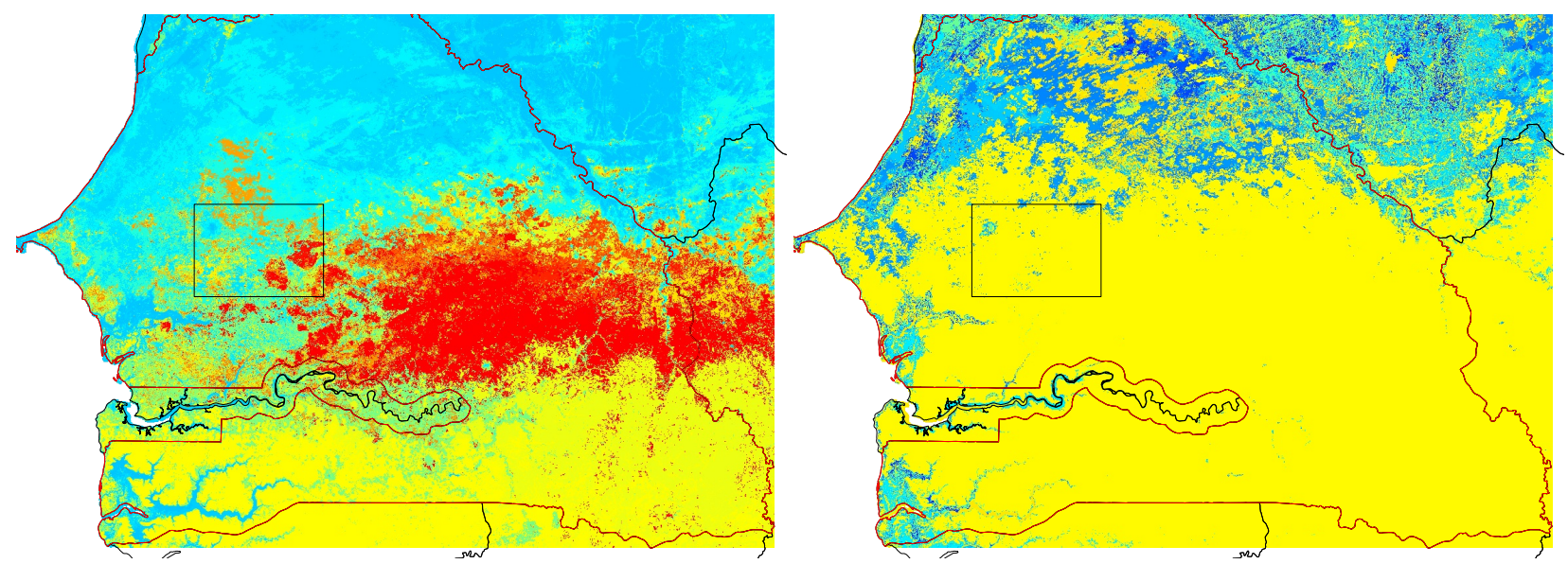

Fig. 6. Final results of the SITS processing with 100 clusters. On the left, clustering of the sequences of typical annual profiles. On the right, clustering of the long time series. The black box locates the region of Touba, Kelkome territory.
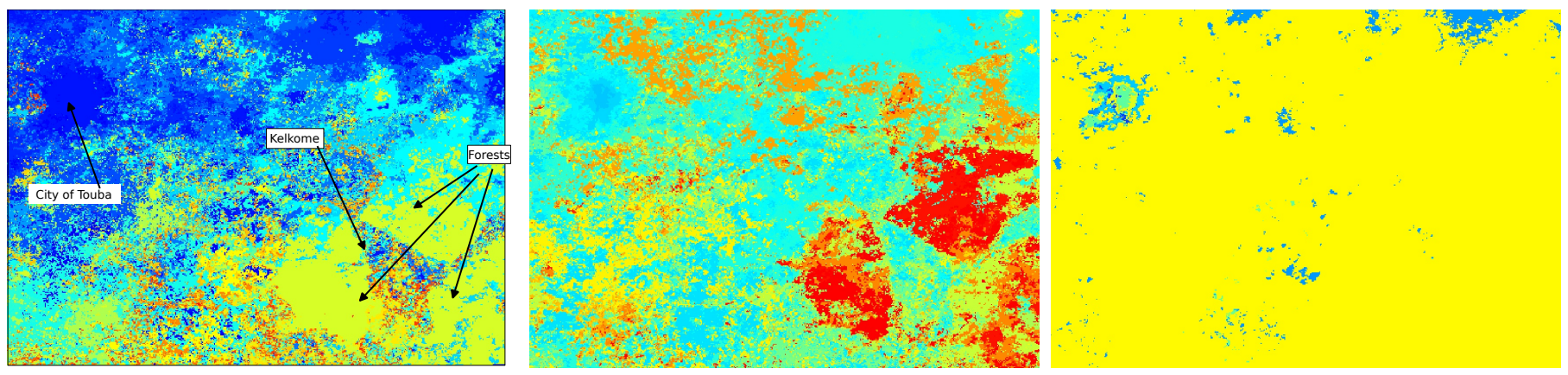

Fig. 7. Clustering of the region between Touba and the territory of Kelkome. (a) Result with clusters constructed only from the pixels of this region, (b) zoom on the image previous clustering image (with cluster from pixels of the whole image), (C) zoom on the image obtained by the clustering of long time series.

characteristic to the vegetation dynamic of these years.

\subsection{Construction of clusters of sequences}

For 30 annual profiles, we get 431717 different sequences. The classification of sequences aims to reduce this number for segmenting the images into regions. The Figure 6 on the left illustrates the results of our method after clustering with 100 clusters. Only 73 clusters are actually present in the image. Figure 6 on the right illustrates results obtained by a clustering of seven years time series. We used K-Means algorithm with $k=100$ and with Euclidean distance between time series.

The result shows that our clustering of sequences of annual profiles better segments the mid-latitudes region of Senegal than the clustering of time series. With the clustering of time series, the whole midlatitude region of Senegal is gathered in a single class. The class distribution shows that this cluster covers more than $66 \%$ of the clustered pixels. This confirms that Euclidean distance on the whole time series does not allow to discriminate the dynamic of the land-cover. However, our two-stages clustering method allows to discriminate two mid-latitudes regions of the Senegal.

Our method also allows to extract fine-grained regions. Around the city of Touba, the territory of Kelkome is an agricultural region (cf. black rectangle in Figure 6 for location). Figure 7 shows three images of this region obtained with different methods. 
The image obtained with the clustering of long time series distinguishes only the city of Touba (low vegetation index all over the years). The zoomed-in image obtained by our method distinguishes the city of Touba and, at the bottom right of images, forests in the neighbourhood of agricultural areas. The last image was obtained by applying our method only on the pixels of this image (with $k=20$ and $l=40$ ). Typical annual profiles and clusters of sequences that were obtained are thus very specific to this region. The resulting clustering is more accurate: forests are more clearly separated from agricultural regions. It should be noticed that our method applied on the whole image has correctly identified some of the fine elements (forests, cities, agricultural region). On the opposite, clustering of long time series makes a lot of confusion between these different land covers.

\section{Conclusion and perspectives}

We presented a clustering method for satellite image time series based on the evolution of vegetation indexes (index $N D V I$ ) on a long term (7 years). The specificity of the proposal stands in a two-stage method: firstly, the construction of typical annual profiles to characterize the behaviour over a period of one year and, secondly, the construction of sequences of typical annual profiles to characterize the behaviour over several years.

This work shows that a multi-temporal scale method provides better results than direct methods. It enabled us to distinguish midlatitude region of Senegal with different behaviour and it extracts the relevant spatial elements in images (cities, forests, agricultural regions).

The vegetation phenology is also an indicator of biological responses to climate change. One of the possible application of this work will be the analysis of the biological responses to climate change at a large spatial scale.

Finally, one of the mid-term goals of this work is to predict soil evolution depending on the precipitation. Our perspective is to use the extracted profiles to accurately predict drought as soon as possible combining information about the multi-annual history of the location and the annual profiles.

\section{References}

Bradley, B.A., Jacob, R.W., Hermance, J.F., Mustard, J.F., 2007. A curve fitting procedure to derive inter-annual phenologies from time series of noisy satellite ndvi data. Remote Sensing of Environment 106, 137-145.

Campbell, J.B., 2002. Introduction to Remote Sensing. The Guilford Press,New York London.

Campo-Bescos, M.A., Munoz-Carpena, R., Southworth, J., Zhu, L., Waylen, P.R., Bunting, E., 2013. Combined spatial and temporal effects of environmental controls on long-term monthly ndvi in the southern africa savanna. Remote Sensing 5, 6513-6538.

Das, G., Gunopoulos, D., Mannila, H., 1997. Finding similar time series, in: Proceedings of the first PKDD Symposium, pp. 88-100.

Diday, E., Murty, N., 2005. Encyclopedia of Data Warehousing and Mining. Idea Group Reference Publisher. chapter Symbolic Data Clustering. pp. $1087-1091$.

Ferretti, A., Prati, C., Rocca, F., 2001. Permanent scatterers in sar interferometry. IEEE Transactions on Geoscience and Remote Sensing 39, 8-20.

Forkel, M., Carvalhais, N., Verbesselt, J., Mahecha, M.D., Neigh, C.S., Reichstein, M., 2013. Trend change detection in ndvi time series: Effects of inter-annual variability and methodology. Remote Sensing 5, 2113-2144.

Julea, A., Méger, N., Bolon, P., Rigotti, C., Doin, M.P., Lasserre, C., Trouvé, E., Lazarescu, V., 2011. Unsupervised spatiotemporal mining of satellite image time series using grouped frequent sequential patterns. Transactions on Geoscience and Remote Sensing 49, 1417-1430.

Kaufman, L., Rousseeuw, P.J., 1990. Finding Groups in Data: An Introduction to Cluster Analysis. John Wiley \& Sons.

Ketterlin, A., Gancarski, P., 2007. Sequence similarity and multi-date image segmentation, in: International Workshop on the Analysis of Multi-temporal Remote Sensing Images, pp. 1-4.

Kuenzer, C., Dech, S., Wagner, W., 2015. Remote Sensing Time Series - Revealing Land Surface Dynamics. Springer

Latecki, L.J., Megalooikonomou, V., Wang, Q., Lakämper, R., Ratanamahatana, C., Keogh, E.J., 2005. Partial elastic matching of time series, in: ICDM'05, pp. 701-704. 
Lezama, J., Alahari, K., Sivic, J., Laptev, I., 2011. Track to the future: Spatio-temporal video segmentation with long-range motion cues, in: IEEE Conference on Computer Vision and Pattern Recognition, p. 0.

Lhermitte, S., Verbesselt, J., Jonckheere, I., Nackaerts, K., van Aardt, J.A., Verstraeten, W.W., Coppin, P., 2008. Hierarchical image segmentation based on similarity of ndvi time series. Remote Sensing of Environment 112, 506-521.

Lhermitte, S., Verbesselt, J., Verstraeten, W., Coppin, P., 2011. A comparison of time series similarity measures for classification and change detection of ecosystem dynamics. Remote Sensing of Environment 115, 3129-3152.

Petitjean, F., Inglada, J., Gançarski, P., 2011. Clustering of satellite image time series under time warping, in: Proceedings of Analysis of Multi-temporal Remote Sensing Images, pp. 69-72.

Petitjean, F., Kurtz, C., Passat, N., Gançarski, P., 2012. Spatio-temporal reasoning for the classification of satellite image time series. Pattern Recogn. Lett. 33, $1805-1815$.

Petitjean, F., Weber, J., 2014. Efficient satellite image time series analysis under time warping. Geoscience and Remote Sensing Letters 11, $1143-1147$.

Sabour, S.T., Lohmann, P., Soergel, U., 2008. Monitoring agricultural activities using multi-temporal asar envisat data, in: Proceedings of The International Archives of the Photogrammetry, Remote Sensing and Spatial Information Sciences, pp. 735-742.

Sakoe, H., Chiba, S., 1978. Dynamic programming algorithm optimization for spoken word recognition. IEEE Transactions on Speech and Signal Processing 26, 43-49.

Trichet, R., Nevatia, R., 2013. Video segmentation with spatio-temporal tubes, in: IEEE International Conference on Advanced Video and Signal Based Surveillance, pp. 330-335.

Tucker, C.J., Vanpraet, C.L., Sharman, M.J., van Ittersum, G., 1985. Satellite remote sensing of total herbaceous biomass production in the senegalese sahel: 1980-1984. Remote Sensing of Environment , 233-249.

Verbesselt, J., Hyndman, R., Newnham, G., Culvenor, D., 2010. Detecting trend and seasonal changes in satellite image time series. Remote Sensing of Environment $114,106-115$. 